\title{
Identification of myeloid derived suppressor cells in the peripheral blood of tumor bearing dogs
}

\author{
Matthew Sherger ${ }^{2}$, William Kisseberth², Cheryl London², Susan Olivo-Marston ${ }^{3}$ and Tracey L Papenfuss ${ }^{\text {* }}$
}

\begin{abstract}
Background: Myeloid derived suppressor cells (MDSCs) are a recently described population of immune cells that significantly contribute to the immunosuppression seen in cancer patients. MDSCs are one of the most important factors that limit the efficacy of cancer immunotherapy (e.g. cancer vaccines) and MDSC levels are increased in cancer in multiple species. Identifying and targeting MDSCs is actively being investigated in the field of human oncology and is increasingly being investigated in veterinary oncology. The treatment of canine cancer not only benefits dogs, but is being used for translational studies evaluating and modifcying candidate therapies for use in humans. Thus, it is necessary to understand the immune alterations seen in canine cancer patients which, to date, have been relatively limited. This study investigates the use of commercially available canine antibodies to detect an immunosuppressive (CD11 $\left.\mathrm{b}^{\text {low }} / \mathrm{CADO} 48^{\text {low }}\right)$ cell population that is increased in the peripheral blood of tumor-bearing dogs.

Results: Commercially available canine antibodies CD11b and CADO48A were used to evaluate white blood cells from the peripheral blood cells of forty healthy control dogs and forty untreated, tumor-bearing dogs.

Tumor-bearing dogs had a statistically significant increase in CD11 blow/CADO48A ${ }^{\text {low }}$ cells (7.9\%) as compared to the control dogs (3.6\%). Additionally, sorted CD11 $\mathrm{b}^{\text {low }} / \mathrm{CADO} 48 \mathrm{~A}^{\text {low }}$ generated in vitro suppressed the proliferation of canine lymphocytes.

Conclusions: The purpose of this study was aimed at identifying potential canine specific markers for identifying MDSCs in the peripheral blood circulation of dogs. This study demonstrates an increase in a unique CD11 b low/ CADO48 $\mathrm{A}^{\text {low }}$ cell population in tumor-bearing dogs. This immunophenotype is consistent with described phenotypes of MDSCs in other species (i.e. mice) and utilizes commercially available canine-specific antibodies. Importantly, CD11 $\mathrm{b}^{\text {low }} / \mathrm{CADO} 48 \mathrm{~A}^{\text {low }}$ from a tumor environment suppress the proliferation of lymphocytes. These results provide a useful phenotype of cells increased in canine cancer patients that may serve as a useful prognostic marker for assessing immune status and functional response to cancer immunotherapies in dogs. Understanding MDSCs in dogs will allow for increased effectiveness of cancer immunotherapy in both dogs and humans.
\end{abstract}

Keywords: Myeloid derived suppressor cells, Cancer immunotherapy, Immunosuppression

\section{Background}

Myeloid derived suppressor cells (MDSC) are immature myeloid cells produced by bone marrow precursor cells that are increased in a variety of diseases. Most significantly, MDSCs are increased in cancer patients and significantly contribute to the immunosuppression of these cancer patients [1]. MDSCs are immature myeloid cells which are arrested during differentiation (myelopoiesis)

\footnotetext{
* Correspondence: papenfuss.1@osu.edu

'Department of Veterinary Biosciences, College of Veterinary Medicine, The Ohio State University, 1900 Coffey Road, Columbus, OH, USA

Full list of author information is available at the end of the article
}

and accumulate $[2,3]$. Utilizing the immune system to specifically target the destruction of cancer cells (i.e. cancer immunotherapy) is conceptually appealing but has proven to be difficult therapeutically. The efficacy of cancer vaccines has proven relatively unsuccessful in patient populations due, in large part, to the dysregulated immune system of cancer patients. MDSCs are significant contributors to the immunosuppression in cancer. Although suppressive myeloid cells were recognized over forty years ago, the understanding that MDSCs contribute to the immunosuppression that limits cancer

\section{Biomed Central}


immunotherapeutics has renewed the interest in these immunosuppressive myeloid cells [4-6].

MDSCs are a heterogeneous population of cells with a variety of phenotypic markers being recognized which have variably been used to identify subsets. By and

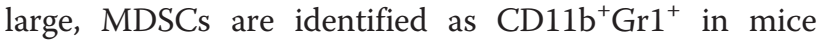
and $\mathrm{CD}^{+} 3^{+} \mathrm{HLA}^{-} \mathrm{DR}^{-} \mathrm{Lin}^{-}$in humans although numerous additional markers (e.g. S100A, etc.) have been used to categorize MDSC subsets. The dual expression of CD11b (myeloid marker) and Gr1 (granulocytic marker) highlights the immature nature of these cells and the fact that these cells arise from a common myeloid precursor that differentiates into dendritic cells (DCs), macrophages and granulocytes. Although present normally at low levels, under pathologic conditions, elevated numbers of these cells are found in both the peripheral circulation and lymphoid organs [7]. The accumulation of MDSCs is thought to be due to a variety of factors including a wide array of soluble factors produced from the tumor environment (e.g. GM-CSF, VEGF, IL-1beta, IL-6, S100A8/A9, etc.). A variety of therapies aimed at limiting MDSC actions have been used to prevent the formation of MDSCs (e.g. tyrosine kinase inhibitors, Sunitinib), reduce MDSC accumulation (e.g. gemcitabine, 5-fluorouracil), affect MDSCs inhibitory abilities (e.g. phosphodiesterase 5 inhibitors, nitroaspirin) or promote MDSC differentiation (e.g. retinoic acid and vitamin D) [1,8-14].

MDSCs suppress both innate and adaptive immune responses through a combination of cell contact-mediated mechanisms (e.g. expression of inhibitory surface marker PD-L1) and the production of a wide array of soluble mediators (e.g. arginase, nitric oxide and reactive oxygen species) $[15,16]$. Cells which have been shown to be inhibited by MDSCs include natural killer (NK) cells, macrophages, DCs, $\mathrm{CD} 4^{+}$helper T cells, $\mathrm{CD}^{+}{ }^{+}$(cytotoxic) T cells and NK-T cells. Additionally, MDSCs are recognized to promote regulatory $\mathrm{T}$ (Treg) cell production where Tregs are potently immunosuppressive in their own right [17]. Through these multiple actions, MDSCs significantly contribute to the immune dysregulation and immunosuppression seen during cancer and are important roadblocks to achieving complete anti-tumor immunity.

Assessment of MDSC levels in cancer patients may provide an important means to evaluate not only relative immune status, but also may be useful biomarkers to evaluate response to therapy. For that reason, easy, rapid and accurate identification of MDSCs is critical. CD11b $\mathrm{br}^{+}$cells are useful markers used to identify MDSCs in mice and studies have demonstrated specific monocytic $\left(\mathrm{Ly} 6 \mathrm{C}^{\text {hi }} \mathrm{Ly}_{6 \mathrm{G}}{ }^{+}\right.$) and granulocytic $\left(\mathrm{Ly} 6 \mathrm{C}^{\mathrm{low}} \mathrm{Ly} 6 \mathrm{G}^{+}\right.$) subsets that may be distinctly contribute to the immunosuppression present in cancer $[1,18,19]$. Similarly, in humans, the $\mathrm{CD}_{3}{ }^{+} \mathrm{HLA}^{-} \mathrm{DR}^{-} \mathrm{Lin}^{-}$MDSC population can be categorized into subpopulations but the contribution of specific subsets to disease pathogenesis is less well defined than in mouse models. The relative identification of MDSCs in other species remains relatively undescribed but has important therapeutic applications in both veterinary and human oncology.

Increasingly, the dog is being used as both a large animal model for carcinogenesis studies and the assessment of cancer therapies due to the similarities between dogs and humans (e.g. outbred population, shared environmental exposures, etc.). Additionally, dog owners are increasingly interested in state-of-the-art therapeutics and are receptive to enrolling their dogs into clinical trials for experimental therapeutics. Thus, there are increasing opportunities not only to assess the responsiveness of canine cancer patients to particular novel therapeutics and cancer immunotherapy, but to apply the acquired information (e.g. drug pharmacokinetics, pharmacodynamics, dosing regimens and efficacy) for clinical applications in human cancer patients. At present, the use of cancer immunotherapy is relatively limited in veterinary medicine but the approval and clinical application of immunotherapies such as the canine melanoma vaccine and Palladia for canine mast cells are reflective of an increased interest in this useful therapeutic approach [9,20. Given these facts, it becomes necessary to understand the immune alterations seen in cancer patients which, to date, have been relatively limited [21,22]. The accurate identification and assessment of MDSC levels in dogs is important to understand the contribution of these potent immunosuppressive cells in canine cancer. Additionally, the identification of an MDSC phenotype provides a means to assess peripheral blood MDSCs which may serve as a useful prognostic marker for assessing immune status and functional response to cancer immunotherapies. This study identified canine specific markers that can be used to identify specific myeloid cell populations within clinical samples from dogs. Our data demonstrate that caninespecific antibodies can be used to identify a specific population of myeloid cells (CD11b ${ }^{\text {low }} C A D O 48 \mathrm{~A}^{\text {low }}$ ) which are increased in tumor-bearing canine patients and that purified CD11b ${ }^{\text {low }}$ CADO48A ${ }^{\text {low }}$ cells suppressed the proliferation of canine lymphocytes.

\section{Methods}

\section{Study Design/Animals}

Eighty client-owned dogs were prospectively enrolled in the study at The Ohio State University's Veterinary Medical Center. Forty dogs presenting to the Community Practice or Blood Bank Services without evidence or history of neoplasia were enrolled into the control group. Forty dogs presenting to the Medical or Radiation Oncology Services were enrolled into the experimental population. Inclusion criteria for patients in the experimental population were (1) cytological or histopatholgic 
diagnosis of a mesenchymal or epithelial neoplasm, (2) local or metastatic disease, (3) no prior surgical of chemotherapeutic treatment and (4) no prior history of neoplasia. No patients with round cell tumors were enrolled in the study. Animal use was approved by The Ohio State University's Institutional Animal Care and Use Committee and The Ohio State University's Veterinary Medical Center's Clinical Research Advisory Committee.

\section{Flow Cytometry Staining and Optimization}

Initial studies optimized the application of commercially available antibodies for detection of MDSCs in dogs. Whole blood samples were obtained through peripheral venipuncture from dogs using approved Institutional Animal Care and Use Committee (IACUC) protocols. The concentration of antibodies and the relative influence of sample handling and influence of time and fixation after staining were evaluated (Figure 1). Briefly, erythrocytes were removed from whole blood samples using erythrocyte lysis buffer $\left(\mathrm{NH}_{4} \mathrm{Cl} / \mathrm{KHCO}_{3} / \mathrm{EDTA}\right)$, washed with $1 \mathrm{x} \mathrm{PBC}$, resuspended in fluorescenceactivated cell sorting buffer (FACS; PBS containing $0.1 \%$ BSA and $0.1 \%$ sodium azide) and placed into flow tubes at a final concentration of $5 \times 10^{5} / 100 \mathrm{uL}$. Cells were then stained with a panel of antibodies including MHC class II (AbdSerotec, MCA1044F; clone YKIX334.2), IgG1 (AbdSerotec, MCA928), CD11b (AbdSerotec, MCA1777S; clone CA16.3E10), CD14 (AbdSerotec, MCA1568A647; clone TUK4), DH59B (previously VMRD, now Monoclonal Antibody Center, Washington State University; clone DH59B), CADO48A (Monoclonal Antibody Center, Washington State University; clone CADO48A) and available isotype controls. All antibodies were primary, non-conjugated with the exception of
MHC class II and CD14 which were directly conjugated to fluorescein isothiocyanate (FITC) and Alexa Fluor 647 (BD Biosciences), respectively. Secondary antibodies of either phycoerythrin (PE; $5 \mathrm{uL}$ ) or Fluorescein isothiocyanate (FITC; 1uL) both from AbD Serotec were used to stain CD11b, CADO48A and DH59B at various dilutions (1:10, 1:50, and 1:100) for optimization studies. Results from these optimization studies were then used on the clinical patient samples. Samples were run on an BD Accuri flow cytometer and analyzed with BD Accuri CFlow analysis software. Samples were run immediately following staining or fixed in $4 \%$ formalin for 4, 24 and 48 hours after staining for optimization (Figure 2) and immediately upon preparation for clinical patient blood samples.

\section{Flow Cytometry of patient samples}

Upon blood collection (EDTA tube), the sample was kept at $4^{\circ} \mathrm{C}$ before staining and all samples were processed within 12 hours of collection. Each patient sample was divided into sample evaluation of 1) cells alone (no antibody), 2) MHC class II, 3) IgG1, 4) CD11b, 5) CADO48A and 6) a combination of CD11b and CADO48A (Figure 3) using antibody concentrations determined during the optimization protocols. The primary antibodies $\mathrm{CD} 11 \mathrm{~b}$ and CADO were secondarily stained with 5uL PE and $1 \mathrm{uL}$ of a 1:50 dilution of FITC respectively. As previously described, samples were prepared and analyzed with the BD Accuri flow cytometer. Data analysis of specific blood cell populations was performed. Specifically, cells were evaluated based on 1) all cells (both dead and live cells, ungated), 2) all peripheral blood mononuclear cells (PBMC) or R1, and 3) all non-lymphocytes based on the appearance of

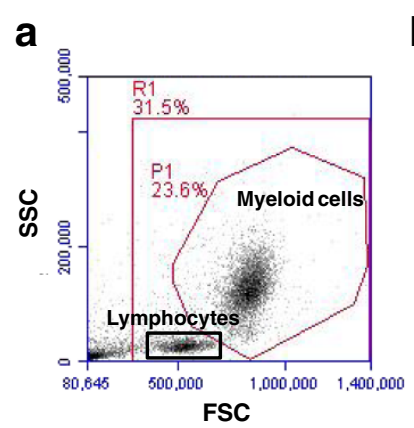

No gate- all cells (live and dead)

R1- all live cells (lymphocytes and myeloid)

P1- all non-lymphocytes

b
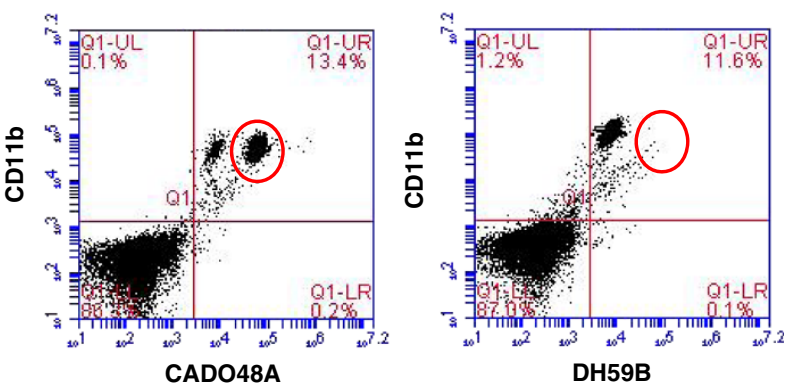

Figure 1 Staining characteristics and evaluation of two commercially available canine granulocytic antibodies of canine peripheral blood. (a) Cells were analyzed according to forward and side scatter. Cells were either gated to include all live and dead (no gate), all live cells including lymphocytes (R1, lymphocytes are circled) and all myeloid cells (P1). (b) Cells were labeled using canine anti-CD1 1b and either (a) CADO48A or (b) DH59B with the same concentration of secondary antibody FITC. As can be seen in (a), CADO48A allows visualization of an additional cell population as compared to DH59B (b). These results were repeatable with multiple blood samples from different dogs. The cells in (b) were gated on P1 as indicated in (a). 


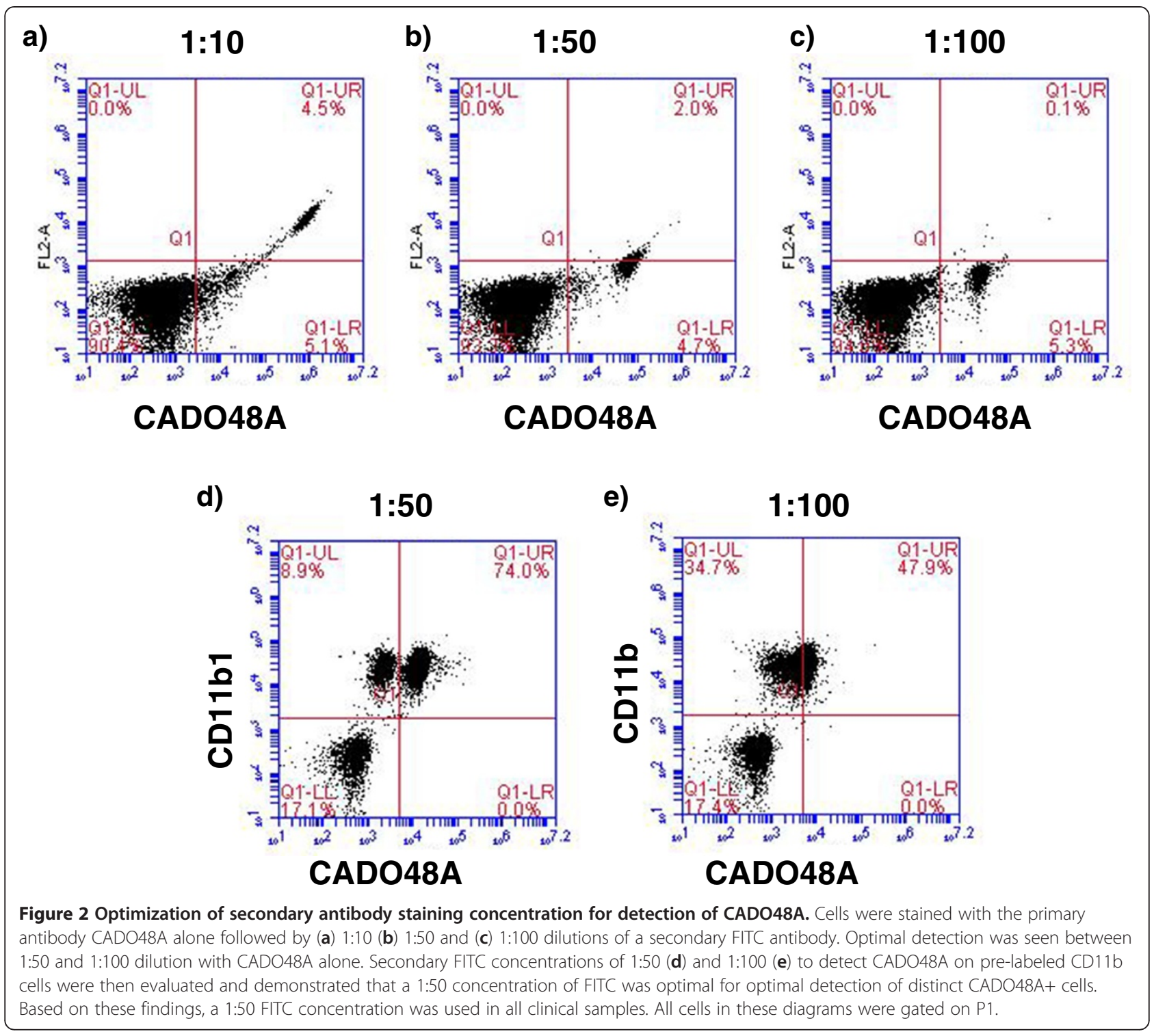

cells on forward scatter (FSC) and side scatter (SSC)

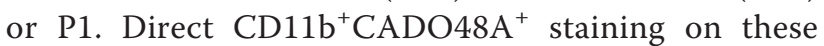
cell populations was assessed (Figure 4). Subsequent gates were assigned as follows P2 $\left(\mathrm{CD} 11 \mathrm{~b}^{\mathrm{hi}} \mathrm{CADO}^{\mathrm{hi}}\right)$, P3 $\left(\mathrm{CD} 11 \mathrm{~b}^{\text {hi }} \mathrm{CADO}^{\text {low }}\right)$ and P4 (CD11b $\left.{ }^{\text {low }} \mathrm{CADO}^{\text {low }}\right)$ (Figure 5). For all gates, the percent and total count of all cells staining positive for both antibodies was determined.

In vitro differentiation, proliferation assay and cytospin of canine MDSCs

Canine bone marrow was approved from humanely euthanized dogs on an approved IACUC protocol. Bone marrow was differentiated in the presence of $10 \mathrm{ng} / \mathrm{ml}$ human GM-CSF (or $20 \mathrm{ng} / \mathrm{ml}$ canine GM-CSF) for 4-5 days with or without $20 \%$ tumor-conditioned media from a canine-specific melanoma MEL-16 line (kindly provided by Dr. Cheryl London). Cells were then labeled as described above for CD11b and CADO48A and sorted using a FACSAris flow sorter. Purified cells were then co-cultured at a 1:5 ratio with responder canine splenocytes and stimulated for 40 hours with $1 \mathrm{ug} / \mathrm{ml} \mathrm{LPS}$ (Sigma) or $3 \mathrm{ug} / \mathrm{ml}$ conA (Sigma) with a final pulse of ${ }^{3} \mathrm{H}$ thymidine in the last 18 hours of culture. An aliquot of cells was prepared by cytospin (1500 rpm for 5 minutes), stained with Wright-Giemsa and photomicrographs taken at a 60x magnification.

\section{Statistical Analysis}

For all statistical analyses, the percentage of cells staining positive for both CADO48A and CD11b were evaluated. Differences between the control and experimental groups were compared using a Wilcoxon rank-sum (Mann-Whitney) test. Additional comparisons between the individual tumor types (sarcoma, carcinoma and 


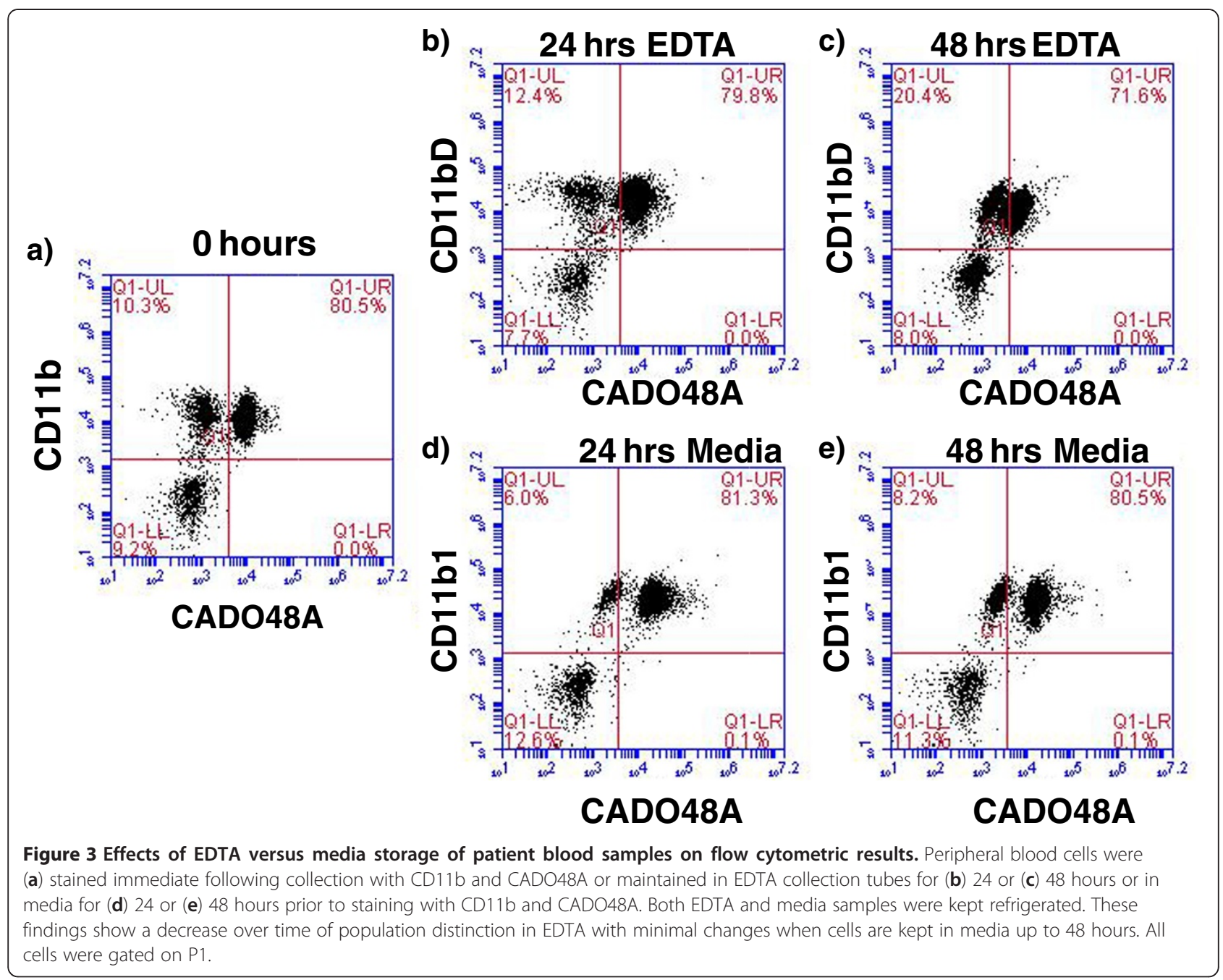

melanoma) were made using a Kruskal-Wallis equalityof-populations rank test. For all comparisons made, p-values less than 0.05 were considered to be significant.

\section{Results}

\section{Flow Cytometry Optimization}

Canine blood samples evaluated by forward scatter (FSC) and side scatter (SSC) (Figure 1) demonstrated distinct populations of small non-granular cells (i.e. lymphocytes) and large granular cells (P1). Based on size and granularity, large granular cells present within the P1 gate were evaluated for expression of cell surface markers of $\mathrm{CD} 11 \mathrm{~b}$ and commercially available canine granulocyte markers CADO48A and DH59B. Figure 1b demonstrates an increased distinction in cell subpopulations evidence with CADO48A staining that was not apparent with DH59B staining. Based on these results, we chose to utilize CADO48A in identifying potential canine MDSCs and myeloid cell populations in canine peripheral blood samples. We first optimized the secondary antibody concentration to detect double positive CD11b and CADO48A. Previous work (data not shown) showed that a 1:50 dilution for secondary antibody staining of CD11b effective identifies $\mathrm{CD}_{11 \mathrm{~b}^{+}}$cells in canine peripheral blood. We next evaluated specific dilutions of secondary FITC antibody staining for detection of CADO48A. Figure 2 shows that a optimal detection was seen at a concentration between 1:50 and 1:100 secondary FITC antibody both in single-labeled $\mathrm{CADO}_{4} 8 \mathrm{~A}^{+}$cells and in cells that were dual-labeled with CD11b on PE and CADO48A on FITC. Based on these findings, a 1:50 FITC concentration was used in all clinical samples. All cells in these diagrams were gated on P1.

Given the variable nature of procurement, handling and processing of clinical samples, we next evaluated the influence of sample handling, timing of antibody labeling and fixation of samples. Figure 3 shows the results of evaluating the effect of immediate staining of cells after collection or staining after storage in either EDTA or 5\% RPMI culture media for 24 or 48 hours. Cells that 


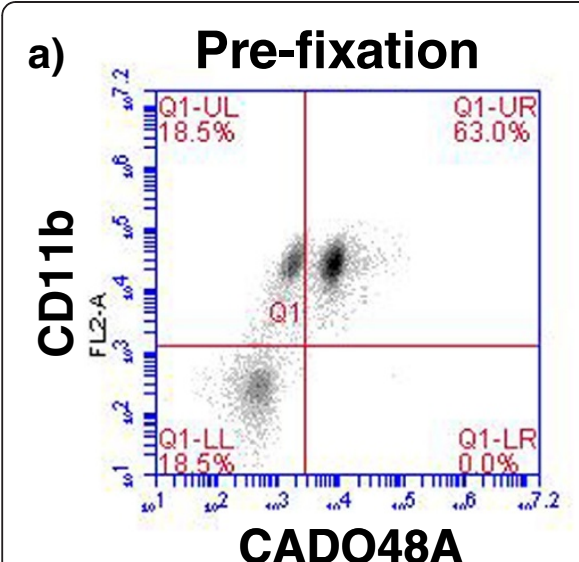

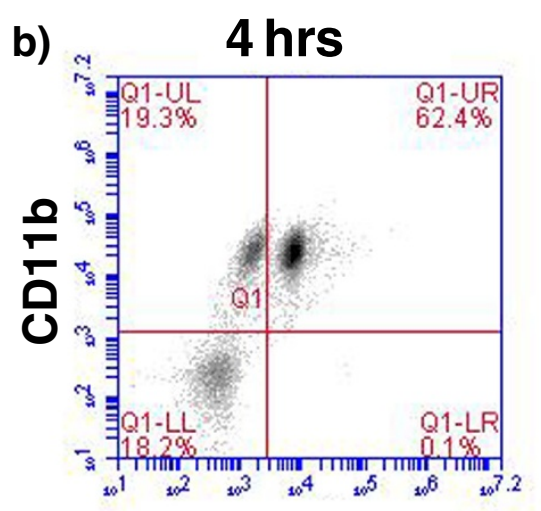

CAD048A

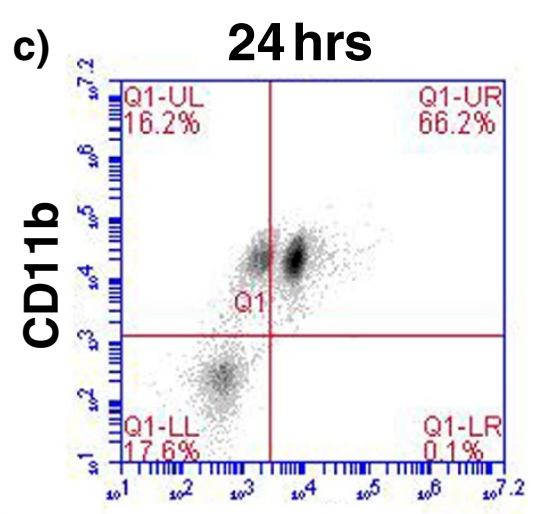

CAD048A
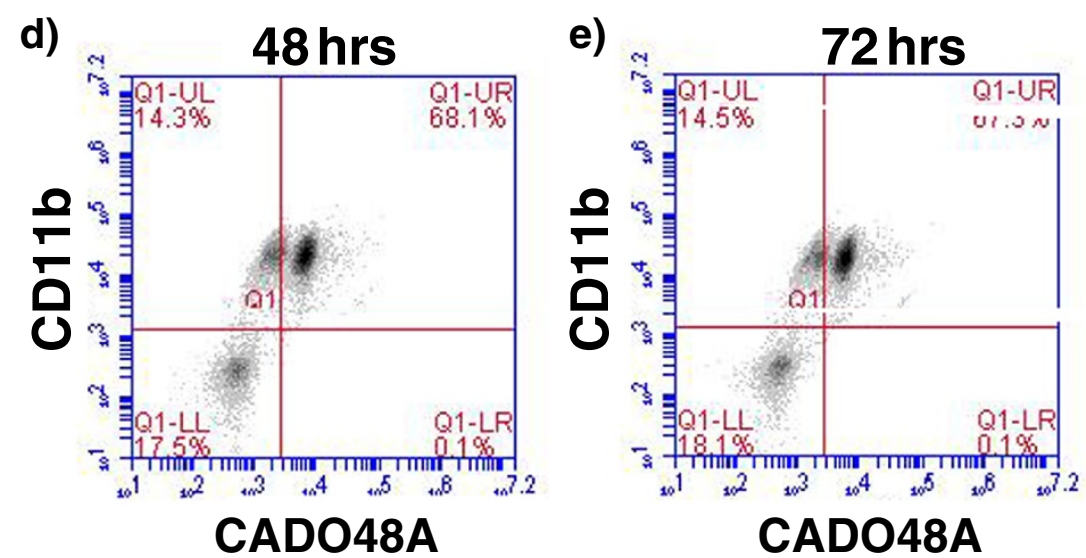

Figure 4 Effections of fixation on CD11b and CADO48A expression levels. Cells were stained for CD11b and CADO48A and evaluated pre-fixation (a) or subsequently fixed with 4\% paraformaldehyde at (b) 4 hours, (c) 24 hours), (d) 48 hours or (e) 72 hours post-fixation before analysis on the flow cytometer. Fixation of cells appears to have limited effects on expression levels of CD11b and CADO48A expression up to 24 hours and then only mild increases of $4-6 \%$ of $C D 11 b^{+} C A D O 48 A^{+}$cells are seen at 24,48 and 72 hours post-fixation. All cells seen in this diagram were gated on P1.
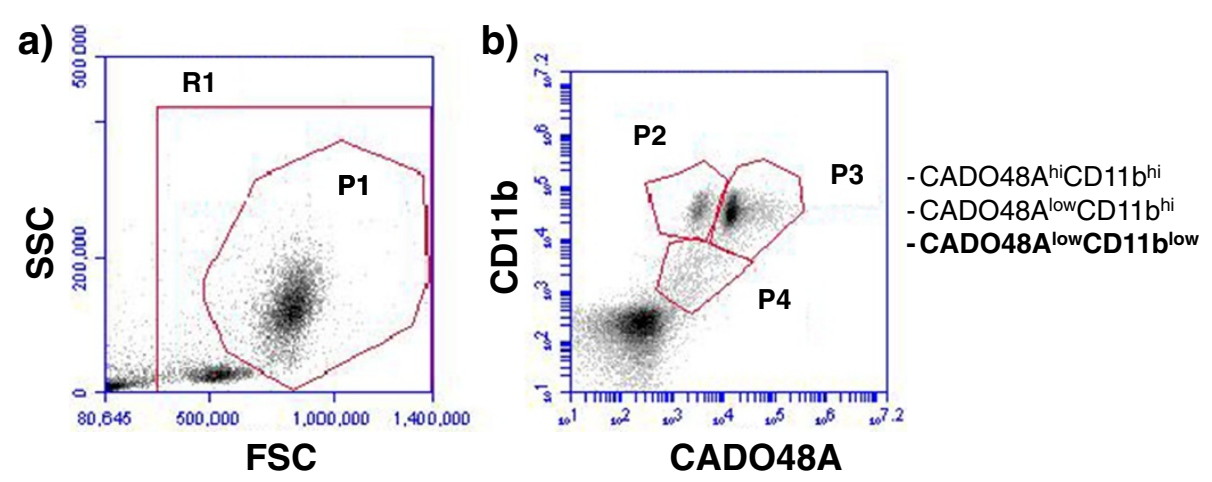

Figure 5 Gated myeloid subpopulations evaluated for patient population. All peripheral blood myeloid cells were evaluated using the P1 gate (non-lymphocytes) and subsequently gated based on relatively expression levels of CD11 b and CADO48A where CADO48A $\mathrm{A}^{\text {hi }} / \mathrm{CD} 11 \mathrm{~b}^{\mathrm{hi}}$ (gate P2), CADO48 $\mathrm{A}^{\text {low }} / \mathrm{CD} 11 \mathrm{~b}^{\text {hi }}$ (gate P3) and CADO48A $\mathrm{A}^{\text {low }} / \mathrm{CD} 11 \mathrm{~b}^{\text {low }}$ (gate P4) likely represent granulocytes/neutrophils, monocytes and MDSCs, respectively. 
were stained immediately upon collection demonstrated the most consistent results compared to those stored in EDTA or media for 24 hours. While cells stored in media demonstrated no change in CD11b/CADO48A expression after 48 hours, there was a slight decrease in $\mathrm{CD}_{11} \mathrm{~b}^{+} \mathrm{CADO} 48 \mathrm{~A}^{+}$cells in cells stored in EDTA for 48 hours. In general, the entire population of cells in 48 hour EDTA-treated cells had diminished CADO48A expression which contributed to the decreased in double positive cells. These findings suggest that media storage of samples for up to 48 hours can provide consistent and similar results to samples processed immediately for evaluated $\mathrm{CD} 11 \mathrm{~b} / \mathrm{CADO} 48 \mathrm{~A}$ expression while storage of samples in EDTA for more than 24 hours may alter the receptor expression of cells. For all subsequent blood sample evaluation, samples were processed immediately unless otherwise indicated.

Fixation of cells with $4 \%$ paraformaldehyde following staining with flow antibodies demonstrated that the expression levels of $\mathrm{CD} 11 \mathrm{~b}$ and $\mathrm{CADO} 48 \mathrm{~A}$ remain relatively constant over time with no change evident in the percentage of $\mathrm{CD} 11 \mathrm{~b}^{+} \mathrm{CADO} 48 \mathrm{~A}^{+}$cells seen even after 72 hours (Figure 4). While immediate evaluation of clinical samples for the expression levels of cell surface markers is ideal, our results suggest that samples can be stored for up to 24 hours in EDTA or 48 hours in media and that cells can be fixed following antibody staining and analyzed up to 72 hours later without dramatically impacting the expression of $\mathrm{CD} 11 \mathrm{~b}^{+} \mathrm{CADO} 48^{+}$levels.

\section{Clinical Patient Blood MDSC Evaluation}

Following the optimization studies, we next evaluated the patient populations of both tumor-bearing and control dogs for the presence of MDSCs. A total of 80 patients were enrolled into the study between April 2011 and January 2012. The control group was comprised of 40 dogs with a median age of 5.0 years (range 315 years) and a variety of breeds represented as outlined in Table 1. The experimental tumor-bearing group was comprised of 40 dogs with a median age of 9.3 years (range 3-14 years) with represented breeds and various tumor types (sarcomas; $n=19$ ), carcinomas; $n=18$ ) and oral melanomas; $n=3$ ) represented (Table 1). The relative expression levels of CD11b and granulocytic markers, such as CADO48A, have been used to identify specific populations of MDSCs. Based on differing levels of both CD11b and CADO48A expression in CD11 ${ }^{+}$CADO48A ${ }^{+}$ cells we found 3 distinct cell populations (P2-P4) that are shown in Figure 6. These cells were CADO48A ${ }^{\text {hi }}$ / $\mathrm{CD}_{11} \mathrm{~b}^{\text {hi }}$ (P2), CADO48A $\mathrm{Al}^{\text {low }} / \mathrm{CD} 11 \mathrm{~b}^{\text {hi }}$ (P3) and CADO $48 \mathrm{~A}^{\text {low }} / \mathrm{CD} 11 \mathrm{~b}^{\text {low }}(\mathrm{P} 4)$ and, based on staining characteristics of $\mathrm{CD} 11 \mathrm{~b}$ and granulocytic marker expression, are most consistent with a neutrophil, monocyte and myeloid precursor (i.e. putative MDSC) population,
Table 1 Description of patient characteristics for the control and experimental groups

\begin{tabular}{|c|c|c|}
\hline Control & & \\
\hline Median Age, years & & 5 \\
\hline Sex & & \\
\hline & Male & 19 \\
\hline & Female & 21 \\
\hline Breed & & \\
\hline & Mixed & 17 \\
\hline & Greyhound & 13 \\
\hline & Labrador Retriever & 3 \\
\hline & Boxer & 2 \\
\hline & Collie & 2 \\
\hline & Other & 3 \\
\hline Tumor & & \\
\hline Median Age, years & & 9 \\
\hline Sex & & \\
\hline & Male & 23 \\
\hline & Female & 17 \\
\hline Breed & & \\
\hline & Lab Retriever & 6 \\
\hline & Mixed & 6 \\
\hline & Greyhound & 4 \\
\hline & Golden Retriever & 4 \\
\hline & Beagle & 3 \\
\hline & Other & 17 \\
\hline Tumor Type & & \\
\hline & Sarcoma & 19 \\
\hline & Carcinoma & 18 \\
\hline & Oral Melanoma & 3 \\
\hline
\end{tabular}

Patient characteristics at the time of study enrollment are described.

respectively. To determine whether specific myeloid cell populations are increased in tumor-bearing dogs, we next evaluated the levels of P2, P3 and P4 in tumorbearing versus control dogs. Table 2 demonstrates the percentage of positive cells and associated statistical parameters (e.g. mean, median, SD, min, max) for both $\mathrm{CD} 11 \mathrm{~b}$ and CADO48A in control and tumor-bearing dogs. While no significant differences were seen in either $\mathrm{P} 2$ or P3, a statistically significant increase in $\mathrm{CD} 11 \mathrm{~b}^{\text {low }}$ / CADO $48 \mathrm{~A}^{\text {low }}$ population (i.e. $\mathrm{P} 4$ gate) was seen $(\mathrm{p}<0.048)$ in tumor-bearing dogs (Table 2, Figure 7). These results show that a CD11b ${ }^{\text {low }} / \mathrm{CADO} 48 \mathrm{~A}^{\text {low }}$ myeloid precursor population was increased in tumor-bearing dogs. We next determined whether tumor-type influenced the levels of CD $11 b^{\text {low }} /$ CADO $48 A^{\text {low }}$ cells. Table 3 demonstrates the percentage of $\mathrm{CD} 11 \mathrm{~b}^{+} \mathrm{CADO} 48 \mathrm{~A}^{+}$cell populations when gated on all live cells (R1), all myeloid non-lymphocyte 

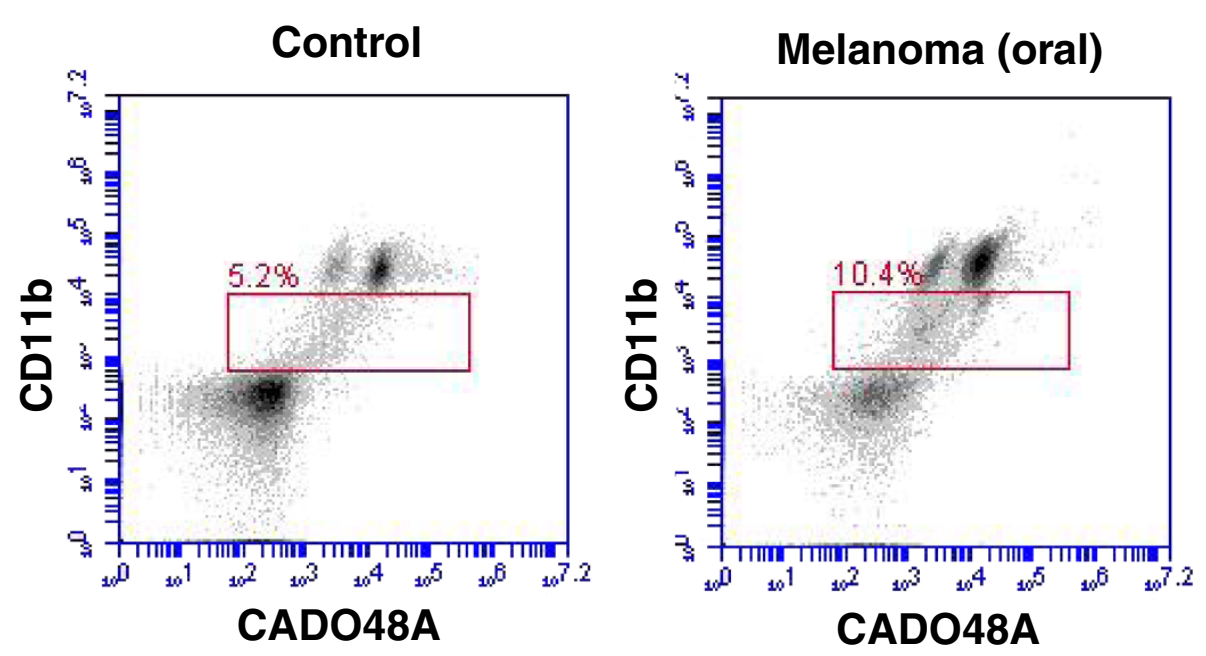

CADO48A
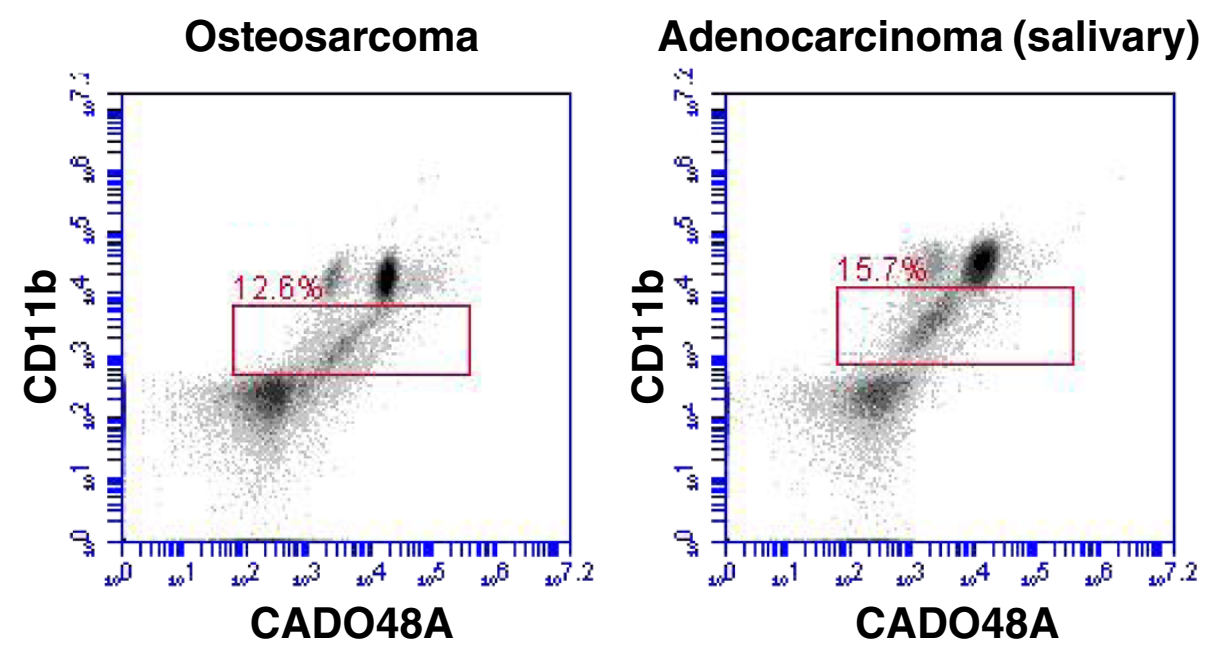

Figure 6 Increased percentage of CD11 $\mathbf{b}^{\text {low }} / C A D O 48 A^{\text {low }}$ in tumor-bearing canine patients. The expression levels of CD $11 b^{\text {low }}$ and CADO48 $A^{\text {low }}$ was evaluated from the peripheral blood of representative canine patients and show an increase of CD $11 b^{\text {low }} / C A D O 48^{\text {low }}$ in tumor-bearing canine patients.

cells (P1) and CD11b/CADO48A subpopulations (P2-P4) across individual tumor types (i.e. sarcoma, carcinoma and melanoma). Table 3 shows an increased percentage of $\mathrm{CD} 1 \mathrm{~b}^{+} \mathrm{CADO} 48 \mathrm{~A}^{+}$cells in both all live cells (i.e. R1 gate, $\mathrm{p}=0.027$ ) and in the myeloid nonlymphocytes (i.e. P1 gate, $\mathrm{p}=0.036$ ). When comparing across tumor types compared to controls with melanomabearing dogs demonstrating the highest levels of R1 and P1. However, an increase in P4 was not consistently evident when samples were grouped on individual tumor-types. Figure 6 demonstrates representative plots of CD11b/CADO48A expression in control dogs and dogs with differing tumor types. Taken together, these data demonstrate that CADO $48 \mathrm{~A}^{\text {low }} / \mathrm{CD} 11 \mathrm{~b}^{\text {low }}$ cells were increased in tumor-bearing dogs and that melanoma-bearing dogs demonstrate the highest levels of

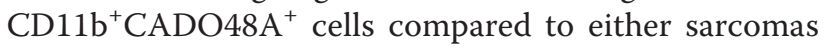
or carcinomas.
Suppressive abilities of CD11 $b^{\text {low }} /$ CADO48A $A^{\text {low }}$ cells

We next wanted to determine whether the increase in $\mathrm{CD}_{11} \mathrm{~b}^{\text {low }} / \mathrm{CADO} 48 \mathrm{~A}^{\text {low }}$ cells present in tumor-bearing dogs could impact immune function. To do this, we generated $\mathrm{CD} 11 \mathrm{~b}^{\text {low }} / \mathrm{CADO} 48 \mathrm{~A}^{\text {low }}$ cells and assessed their ability to suppress the proliferation of responder canine immune cells. To generate sufficient numbers of $\mathrm{CD} 11 \mathrm{~b}^{\text {low }} /$ CADO48A ${ }^{\text {low }}$ cells for functional evaluation, we utilized an in vitro system to differentiate canine MDSCs and control myeloid precursor cells from bone marrow myeloid progenitors. Sorted CD $11 b^{\text {low }} / \mathrm{CADO} 48 \mathrm{~A}^{\text {low }}$ cells were able to suppress the proliferation of responder canine splenocytes following conA stimulation but not in LPS-stimulated cells (Figure 7). Cellular morphology was relatively similar between control and melanoma myeloid precursors although overall expression levels of both CD11b and CADO48A was slightly decreased in the myeloid cells exposed to melanoma tumor-conditioned media which 
Table 2 Comparison of percent of cells positive for CD11b/CADO48A by gate of control and tumor-bearing dogs

\begin{tabular}{lrrrr}
\hline Control & Mean & Median & Min & Max \\
\hline Ungated & $31.9 \pm 14.4$ & 27.2 & 12.6 & 58.2 \\
R1 & $28.4 \pm 10.3$ & 24.4 & 10.9 & 50.6 \\
P1 & $27.5 \pm 10.1$ & 23.6 & 10.6 & 49.0 \\
P2 & $24.2 \pm 09.2$ & 20.8 & 8.6 & 45.3 \\
P3 & $4.6 \pm 2.8$ & 4.0 & 1.5 & 13.4 \\
P4 & $3.6 \pm 1.7^{*}$ & 3.0 & 1.3 & 7.7 \\
Tumor & & & & \\
Ungated & $33.7 \pm 15.0$ & 29.3 & 11.4 & 72.3 \\
R1 & $30.0 \pm 14.1$ & 26.9 & 8.5 & 66.9 \\
P1 & $29.1 \pm 14.0$ & 26.0 & 7.2 & 65.2 \\
P2 & $25.5 \pm 12.8$ & 22.5 & 8.1 & 59.6 \\
P3 & $4.5 \pm 3.6$ & 3.5 & 1.2 & 20.3 \\
P4 & $7.9 \pm 3.0^{*}$ & 3.7 & 1.1 & 12.7 \\
\hline
\end{tabular}

The percentage of cells staining positive for CD11b and CADO48A are displayed for each gate analyzed for both the control and tumor-bearing populations. Cells from the P4 gate of the tumor bearing dogs were found to be elevated as compared to the healthy control dogs. No other statistical differences were seen in the other gates analyzed. An* denotes statistical significance $(P<0.05)$. All values are expressed as a percentage of all cells analyzed by flow cytometry.

is consistent with the relative arrest in differentiation described for MDSCs. Taken together, these results

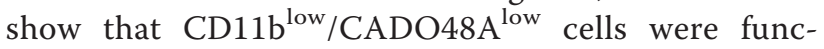
tionally immunosuppressive and likely represent a canine MDSC population.

\section{Discussion}

This study investigated potential canine-specific markers for identifying MDSCs in the peripheral blood of dogs with cancer. We began our studies by first identifying potential phenotypic markers that could be used for identifying canine MDSCs. Prototypic MDSC phenotypic markers have been reported as $\mathrm{CD} 11 \mathrm{~b}^{+} / \mathrm{CD} 33^{+} / \mathrm{HLDR}^{\text {low }}$

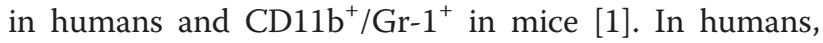
CD15 (granulocytic) and CD14 (monocytic) identify subpopulations of these cells while, in mice, $\mathrm{CD} 11 \mathrm{~b}^{+} \mathrm{Ly}_{6 \mathrm{G}}{ }^{+}$and $\mathrm{CD} 11 \mathrm{~b}^{+} \mathrm{Ly}_{6 \mathrm{C}^{+}}$cells identify these cells as either granulocytic or monocytic, respectively. Unfortunately, the lack of readily available commercial canine antibodies has limited the identification of MDSCs and MDSC subsets in dogs.

$\mathrm{CD} 11 \mathrm{~b}$, an integrin, is found on a variety of cells of myeloid origin and has been used as one phenotypic marker of MDSCs present in the mouse and man. In the dog, CD11b in known to be a marker of cells of myeloid origin, most specifically neutrophils [23]. There is a commercially available anti-canine $\mathrm{CD} 11 \mathrm{~b}$ available which has been validated. In the mouse, Gr-1 is a granulocytic marker whose co-expression with CD11b is used to identify the immature myeloid cell population that represents MDSCs. Although CD11b is useful to identify myeloid (e.g. macrophages/DCs), neutrophils and mast cells in the dog, no Gr-1 equivalent for the dog has been described. Additionally, murine $\mathrm{Gr}-1$ has demonstrated a variable ability to stain canine cells, and its individual components, Ly6G and Ly6C, are not reported to cross-react (unpublished observations) $[21,24,25]$.

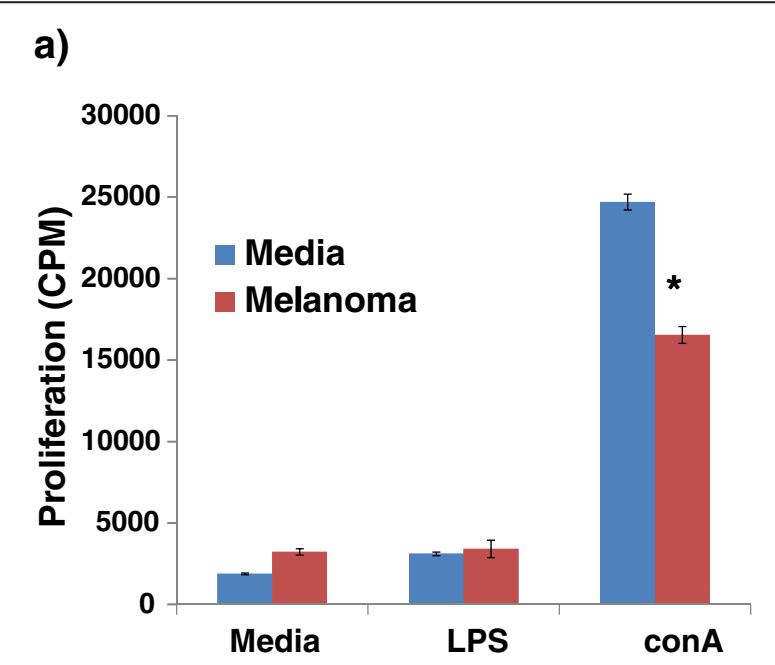

b)

b) Media

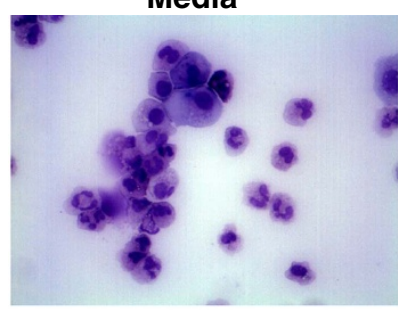

Melanoma

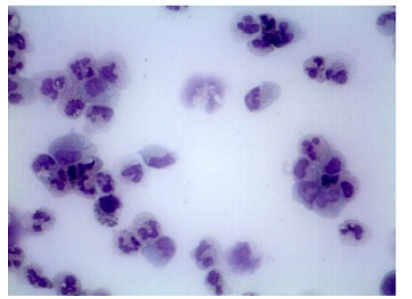

Figure 7 Purified $C D 11 b^{\text {low }}$ CADO48A $A^{\text {low }}$ cells from in vitro differentiated myeloid cells suppressed the proliferation of $T$ and $B$ lymphocytes. Bone marrow cells were differentiated in either GM-CSF (Media) or GM-CSF with canine melanoma tumor-conditioned media for 5 days. Cells were purified and co-cultured at a 1:5 ratio with responder canine splenocytes and the (a) proliferation of cells determined. A cytospin (b) of purified cells shows a mixed population of myeloid and granulocytic precursor cells demonstrating monocytic, granulocytic and ring-shaped nuclei are present in the $\mathrm{CD} 11 \mathrm{~b}^{\text {low/ }} \mathrm{CADO} 48 \mathrm{~A}^{\text {low }}$ populations of both media and melanoma cells. Photomicrograph of the cytospin is at 60x magnification. 
Table 3 Comparison of percent of cells positive for CD11b/CAD048A by gate amongst tumor types

\begin{tabular}{|c|c|c|c|c|}
\hline Sarcoma & Mean & Median & Min & Max \\
\hline Ungated & $33.4 \pm 12.7$ & 28.7 & 13.5 & 58.2 \\
\hline $\mathrm{R} 1$ & $29.9 \pm 12.5^{*}$ & 26.5 & 11.7 & 53.1 \\
\hline P1 & $29.0 \pm 12.6^{*}$ & 25.5 & 11.6 & 51.8 \\
\hline $\mathrm{P} 2$ & $25.1 \pm 12.0$ & 22.4 & 8.2 & 50.6 \\
\hline P3 & $4.8 \pm 4.7$ & 3.5 & 1.6 & 20.3 \\
\hline P4 & $4.7 \pm 2.9$ & 3.8 & 1.1 & 12.6 \\
\hline \multicolumn{5}{|c|}{ Carcinoma } \\
\hline Ungated & $30.4 \pm 14.8$ & 23.8 & 11.4 & 64.5 \\
\hline $\mathrm{R} 1$ & $26.4 \pm 12.8^{*}$ & 21.5 & 8.5 & 55.6 \\
\hline P1 & $25.3 \pm 12.5^{*}$ & 21.0 & 7.2 & 53.6 \\
\hline $\mathrm{P} 2$ & $22.7 \pm 11.4$ & 18.2 & 8.1 & 47.9 \\
\hline P3 & $3.7 \pm 1.9$ & 3.1 & 1.2 & 8.0 \\
\hline P4 & $5.1 \pm 3.5$ & 3.5 & 2.1 & 12.7 \\
\hline \multicolumn{5}{|c|}{ Melanoma } \\
\hline Ungated & $56.0 \pm 15.2$ & 53.5 & 42.3 & 72.3 \\
\hline $\mathrm{R} 1$ & $52.6 \pm 13.5^{*}$ & 51.0 & 40.0 & 66.9 \\
\hline P1 & $51.6 \pm 13.0^{*}$ & 50.2 & 39.3 & 65.2 \\
\hline P2 & $44.7 \pm 13.5$ & 41.2 & 33.3 & 59.6 \\
\hline P3 & $7.8 \pm 2.2$ & 7.7 & 5.7 & 10.1 \\
\hline P4 & $4.8 \pm 1.1$ & 4.5 & 3.8 & 6.0 \\
\hline
\end{tabular}

The percentage of cells staining positive for CD11b and CADO48A are displayed for each tumor subtype: sarcoma $(n=19)$, carcinoma $(n=18)$ and melanoma $(n=3)$. Patients were categorized into one of the three groups based on either cytology or histopathology. A statistical difference was seen in the R1 and P1 gates between the 3 tumor types on initial analysis. When the melanoma patients were excluded due to low patient numbers no statistical difference was seen in the percent of CD11b/CADO48A positive cells, in any gate, between the sarcoma or carcinoma patients. An* denoted statistical significance $(p<0.05)$. All values are expressed as a percentage of all cells analyzed by flow cytometry.

In man, an equivalent antibody to $\mathrm{Gr}-1$ does not exist. Rather, CD33, CD14 and HLA-DR (i.e. MHC class II) expression are used to specifically identify these cells. No canine specific, or cross-reacting, CD33 antibody is currently commercially available. Although human CD14 cross-reactive with canine cells, canine CD14 expression is variable on monocytes and likely may not truly mimic what is seen in humans [26]. Additionally, standardized protocols and antibody concentrations using CD14 in the canine have not been established [27]. However, a recent publication by Goulart et al. has demonstrated the use of negative labeling for CD14 expression to identify an MDSC population in the dog but no studies have investigated canine-specific granulocytic antibodies to identify an MDSCs in the dog [21]. Considering the lack of anti-canine CD33 and human MDSC subpopulations express markers which are not commercially available (e.g. canine-specific CD15 or CD14), we chose to evaluate the levels of canine-specific $\mathrm{CD} 11 \mathrm{~b}$ and a granulocytic marker which models the phenotypic description for murine MDSCs.

Only two commercially available markers are reported to label canine granulocytes (i.e. CADO48A and DH59A) and our results show that CADO48A is able to distinguish separate populations that are not evident in DH59A stained populations from canine peripheral blood. While our focus was on canine-specific antibodies, we did evaluate the cross-reactivity of murine $\mathrm{Gr}-1$ with canine peripheral blood samples. Murine Gr-1 (clone RB6-8C5) failed to stain canine peripheral blood cells, results which differ from the ability of this clone to stain canine cells by Goulart et al. [21]. Our studies suggest that canine-specific CADO48A is an effective antibody to identify circulating myeloid cells in canine blood.

The ability of CADO48A to identify individual granulocytic populations of peripheral white blood cells (high versus low expression) as seen in Figure 1 suggests that this antibody may be useful for identifying granulocytic versus monocytic subpopulation but purification and functional evaluation of such subpopulations were beyond the scope of this study. Our results demonstrate that CD11b and CADO48 staining is useful for detecting subpopulations of myeloid cells in the peripheral blood of dogs. From our optimization studies, we found that clinical samples can be stored up to 24 hours in EDTA, 48 hours in media and that cells that have been fixed following staining with flow antibodies can be analyzed up to 72 hours later without dramatically impacting

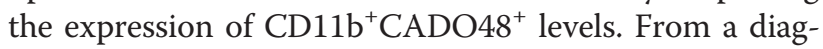
nostic standpoint, it is important to know the relative expression levels of these markers depending on sample treatment and handling.

Following optimization, we next went on to determine whether tumor-bearing dogs demonstrated altered levels of myeloid cell populations. Our results show that a specific population of $\mathrm{CD} 11 \mathrm{~b}^{\text {low }} \mathrm{CADO} 48 \mathrm{~A}^{\text {low }}$ cells was increased in tumor-bearing dogs. The finding of an upregulated population of cells in tumor-bearing dogs expressing CD11b, as well as, the surrogate marker CADO48A, is suggestive of an MDSC phenotype in the canine. Classically, MDSC expression of CD11b is considered to be high. In our canine samples, we found that the expression in the canine patient to be low. While this finding is discordant from the literature on MDSCs in the mouse and human, CD11b expression is known to be variable both in healthy people at different time points and in animals with varying severity of inflammation $[28,29]$. Additionally, it has been shown in human cell lines that during neutrophil maturation from a promyelocytic stage of development to a more terminally differentiated state CD11b expression starts low and eventually reaches high levels of expression once fully mature [30]. If the cells in our tumor-bearing dogs 
represent an immature myeloid population, low $\mathrm{CD} 11 \mathrm{~b}$ expression may be representative of MDSCs. Additionally, work done by Furuhashi et al. has shown that DCs with high CD11b expression are more capable of eliciting a $\mathrm{T}$-cell response in the pulmonary parenchyma of mice, indicating that increased $\mathrm{CD} 11 \mathrm{~b}$ expression is a relative marker of increased maturation and/or activation of DCs [31]. Based on our results and previously published findings, low CD11b expression may in fact be indicative of an early myeloid derived cell population such as MDSC. Additionally, the cellular morphology of our purified $\mathrm{CD}_{11} \mathrm{~b}^{\text {low }} \mathrm{CADO} 48 \mathrm{~A}^{\text {low }}$ cells is consistent with an immature and heterogeneous cellular morphology described for MDSC populations $[1,18,19]$ in mice which contain cells demonstrating monocytic, granulocytic and ring-shaped nuclear forms (Figure 7B). Thus, we postulated that CD11b ${ }^{\text {low }}$ CADO $48 \mathrm{~A}^{\text {low }}$ represent an MDSC population.

In order to determine whether, indeed, $\mathrm{CD} 11 \mathrm{~b}^{\text {low }}$ CADO48 $\mathrm{A}^{\text {low }}$ were immunosuppressive, we utilized our in vitro model to generate canine MDSCs under the influence of a tumor environment and purified the CD11b ${ }^{\text {low }}$ CADO48A ${ }^{\text {low }}$ population. As Figure 7 demonstrates, $\mathrm{CD} 11 \mathrm{~b}^{\text {low }} \mathrm{CADO} 48 \mathrm{~A}^{\text {low }}$ were able to suppress the proliferation of responder canine lymphocytes. While cell sorting and functional evaluation of individual cancer patients were beyond the scope of this study, our data from purified $\mathrm{CD} 11 \mathrm{~b}^{\text {low }} \mathrm{CADO} 48 \mathrm{~A}^{\text {low }}$ (from control or tumor environment) verified that $\mathrm{CD} 11 \mathrm{~b}^{\text {low }} \mathrm{CADO} 48 \mathrm{~A}^{\text {low }}$ were immunosuppressive. Ongoing studies are evaluating the functional abilities and signaling pathways involved in the development of canine MDSCs using our in vitro MDSCs model.

While significant differences in R1 and P1 were found when tumor-bearing patients were grouped according to tumor type (Table 3), it was somewhat surprising that $\mathrm{P} 4$ differences were not significant. The most likely reason for this is that subcategorization decreased the number of patients in each tumor type category which then resulted in a lack of statitistical significance. An additional prospective study with recruitment of more patients according to individual tumor types would likely address this possibility. One interesting aspect of our study is the mixed population of tumors evaluated. In the human literature, the predominance of tumors shown to upregulate MDSCs are carcinomas, including those arising from the pancreas, colon and lung [32-34]. Unlike in humans, sarcomas are a relatively common tumor type seen in veterinary species and studies investigating MDSC populations in humans have found this cell type primarily in various carcinomas and melanoma [35]. The fact that MDSCs were upregulated in our tumor-bearing population, regardless of tumor type suggests that common mechanisms exist between sarcomas and carcinomas for the induction of MDSCs in the canine population. Interestingly, melanomas appear to upregulate MDSCs regardless of species (Table 3) [32-35]. Studies of MDSCs in dogs may be useful at dissecting the potential mechanisms by which carcinomas, sarcomas or melanomas differentially regulate MDSCs levels and why these may differ between species. Additionally, specific tumor types may uniquely upregulate specific MDSC subsets such as seen with the predominance of $\mathrm{CD} 15^{+}$MDSC or lineage negative MDSCs in patients with glioblastoma [36]. At present, a detailed evaluated of canine MDSC subpopulations is not feasible but our data suggest that CADO48A may be a useful antibody for such distinctions.

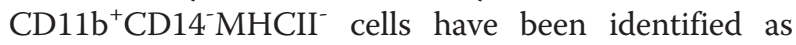
MDSCs in the peripheral blood of dogs (Goulart 2012) in both solitary and metastatic cancer and these cells were able to suppress $\mathrm{T}$ cell proliferation similar to what we found with $\mathrm{CD} 11 \mathrm{~b}^{\text {low }} \mathrm{CADO} 48 \mathrm{~A}^{\text {low }}$ cells [115]. Our studies demonstrate that CADO48A is a useful marker to identify MDSCs in dogs and it is not known whether

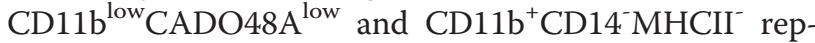
resent two distinct MDSC populations (e.g. granulocytic and monocytic, respectively) and, if so, whether these two populations have distinct functional profiles. The identification of MDSC subpopulations is likely to be useful for diagnostic and prognostic purposes but the characterization of these populations in canines is only in its infancy. Additional markers, such as S100A9, have been described to identify monocytic MDSCs in humans with colon cancer and cross-reacting S100A9 proteins are available [33]. Ongoing studies evaluating various MDSC markers are needed to determine the phenotype and function of specific MDSC subpopulations (e.g. monocytic and granulocytic) in dogs. Utilization of both canine patients and an in vitro model system as we provide here are likely to provide important data on MDSC phenotype and function and facilitate translational applications between canine and human MDSC studies.

\section{Conclusion}

This study identified canine specific markers that can be used to identify specific myeloid cell populations within clinical samples from dogs. Our data demonstrate that canine-specific antibodies can be used to identify a specific population of myeloid cells $\left(\mathrm{CD} 11 \mathrm{~b}^{\text {low }} \mathrm{CADO} 48 \mathrm{~A}^{\text {low }}\right.$ ) which are increased in tumor-bearing canine patients and that purified $\mathrm{CD} 11 \mathrm{~b}^{\text {low }} \mathrm{CADO} 48 \mathrm{~A}^{\text {low }}$ cells suppressed the proliferation of canine lymphocytes. This work provides a foundation for future investigations of MDSC levels which may serve as a prognostic indicator in canine patients and help guide translational research approaches for cancer immunotherapy in human and veterinary cancer patients. The field of immunotherapy, both in man and in the veterinary field, is in a state of constant discovery. The ability to identify and monitor MDSC levels in the 
dog will be useful in the development and evaluation of new therapies in both man and his best friend.

\section{Competing interests}

The authors declare that they have no competing interests that could inappropriately influence or bias the content of this paper.

\section{Authors' contribution}

MS assisted in the design of the study, conducted the experiments, interpreted the data and drafted the manuscript. WK participated in study design and helped to draft the manuscript. CL participated in study design and helped to draft the manuscript. SOM performed statistical analysis. TP conceived the study, participate in its design and helped to draft the manuscript. All authors read and approved the final manuscript.

\section{Acknowledgments}

This work was supported by an intramural canine research grant from the College of Veterinary Medicine of The Ohio State University.

\section{Author details}

'Department of Veterinary Biosciences, College of Veterinary Medicine, The Ohio State University, 1900 Coffey Road, Columbus, OH, USA. ${ }^{2}$ Department of Veterinary Clinical Sciences, College of Veterinary Medicine, The Ohio State University, 1900 Coffey Road, Columbus, OH, USA. ${ }^{3}$ Division of Epidemiology, College of Public Health, The Ohio State University, 1841 Neil Avenue, Columbus, OH, USA

Received: 22 May 2012 Accepted: 24 October 2012

Published: 31 October 2012

\section{References}

1. Gabrilovich DI, Nagaraj S: Myeloid-derived suppressor cells as regulators of the immune system. Nat Rev Immunol 2009, 9(3):162-174.

2. Youn Jl, Gabrilovich DI: The biology of myeloid-derived suppressor cells: the blessing and the curse of morphological and functional heterogeneity. Eur J Immunol 2010, 40(11):2969-2975.

3. Medzhitov R, et al: Highlights of 10 years of immunology in Nature Reviews Immunology. Nat Rev Immunol 2011, 11(10):693-702.

4. Krystal $\mathrm{G}$, et al: Re: the terminology issue for myeloid-derived suppressor cells. Cancer Res 2007, 67(8):3986.

5. Kirchner $\mathrm{H}$, et al: Evidence of suppressor cell activity in spleens of mice bearing primary tumors induced by Moloney sarcoma virus. J Exp Med 1974, 139(6):1473-1487.

6. Gabrilovich Dl, et al: Dendritic cells in antitumor immune responses. II. Dendritic cells grown from bone marrow precursors, but not mature DC from tumor-bearing mice, are effective antigen carriers in the therapy of established tumors. Cell Immunol 1996, 170(1):111-119.

7. Diaz-Montero $C M$, et al: Increased circulating myeloid-derived suppresso cells correlate with clinical cancer stage, metastatic tumor burden, and doxorubicin-cyclophosphamide chemotherapy. Cancer Immunol Immunother 2009, 58(1):49-59.

8. Ko JS, et al: Sunitinib mediates reversal of myeloid-derived suppressor cell accumulation in renal cell carcinoma patients. Clin Cancer Res 2009, 15(6):2148-2157

9. London CA: Tyrosine kinase inhibitors in veterinary medicine. Top Companion Anim Med 2009, 24(3):106-112.

10. Ugel S, et al: Therapeutic targeting of myeloid-derived suppressor cells. Curr Opin Pharmacol 2009, 9(4):470-481.

11. Serafini P, Borrello I, Bronte V: Myeloid suppressor cells in cancer: recruitment, phenotype, properties, and mechanisms of immune suppression. Semin Cancer Biol 2006, 16(1):53-65.

12. Shojaei $F$, et al: Tumor refractoriness to anti-VEGF treatment is mediated by CD11b+Gr1+ myeloid cells. Nat Biotechnol 2007, 25(8):911-920.

13. Fujita $\mathrm{M}$, et al: COX-2 blockade suppresses gliomagenesis by inhibiting myeloid-derived suppressor cells. Cancer Res 2011, 71(7):2664-2674.

14. Bunt SK, et al: Reduced inflammation in the tumor microenvironment delays the accumulation of myeloid-derived suppressor cells and limits tumor progression. Cancer Res 2007, 67(20):10019-10026.

15. Ilkovitch D, Lopez DM: The liver is a site for tumor-induced myeloidderived suppressor cell accumulation and immunosuppression. Cancer Res 2009, 69(13):5514-5521.
16. Talmadge JE: Pathways mediating the expansion and immunosuppressive activity of myeloid-derived suppressor cells and their relevance to cancer therapy. Clin Cancer Res 2007, 13 (18 Pt 1):5243-5248.

17. Pan PY, et al: Immune stimulatory receptor CD40 is required for T-cell suppression and T regulatory cell activation mediated by myeloidderived suppressor cells in cancer. Cancer Res 2010, 70(1):99-108.

18. Ostrand-Rosenberg S: Myeloid-derived suppressor cells: more mechanisms for inhibiting antitumor immunity. Cancer Immunol Immunother 2010, 59(10):1593-1600.

19. Ostrand-Rosenberg S, Sinha P: Myeloid-derived suppressor cells: linking inflammation and cancer. J Immunol 2009, 182(8):4499-4506.

20. Bergman PJ, et al: Development of a xenogeneic DNA vaccine program for canine malignant melanoma at the Animal Medical Center. Vaccine 2006, 24(21):4582-4585.

21. Goulart MR, Pluhar GE, Ohlfest JR: Identification of myeloid derived suppressor cells in dogs with naturally occurring cancer. PLoS One 2012, 7(3):e33274.

22. Mitchell L, Thamm DH, Biller BJ: Clinical and immunomodulatory effects of toceranib combined with low-dose cyclophosphamide in dogs with cancer. J Vet Intern Med 2012, 26(2):355-362.

23. Kobayashi T, et al: Canine hair-follicle keratinocytes enriched with bulge cells have the highly proliferative characteristic of stem cells. Vet Dermatol 2009, 20(5-6):338-346.

24. Mazzone A, et al: Correlation between CD11b/CD18 and increase of aggregability of granulocytes in coronary artery disease. Inflammation 1992, 16(4):315-323.

25. Lin $T Y$, Rush $\sqcup$, London CA: Generation and characterization of bone marrow-derived cultured canine mast cells. Vet Immunol Immunopathol 2006, 113(1-2):37-52.

26. Pinet $F$, et al: Morphology, homogeneity and functionality of human monocytes-derived macrophages. Cell Mol Biol (Noisy-le-grand) 2003, 49(6):899-905

27. Schuberth $\mathrm{HJ}$, et al: Reactivity of cross-reacting monoclonal antibodies with canine leukocytes, platelets and erythrocytes. Vet Immunol Immunopathol 2007, 119(1-2):47-55.

28. Rebuck N, Finn A: Polymorphonuclear granulocyte expression of CD11a/ CD18, CD11b/CD18 and L-selectin in normal individuals. FEMS Immunol Med Microbiol 1994, 8(3):189-195.

29. Dosogne $H$, et al: Increased surface expression of CD11b receptors on polymorphonuclear leukocytes is not sufficient to sustain phagocytosis during Escherichia coli mastitis in early postpartum dairy cows. Vet Immunol Immunopathol 1997, 60(1-2):47-59.

30. Chang $\mathrm{HH}$, et al: Multistable and multistep dynamics in neutrophil differentiation. BMC Cell Biol 2006, 7:11.

31. Furuhashi $\mathrm{K}$, et al: Mouse lung CD103+ and CD11bhigh dendritic cells preferentially induce distinct CD4+ T-cell responses. Am J Respir Cell Mol Biol 2012, 46(2):165-172.

32. Porembka MR, et al: Pancreatic adenocarcinoma induces bone marrow mobilization of myeloid-derived suppressor cells which promote primary tumor growth. Cancer Immunol Immunother 2012,

33. Zhao F, et al: S100A9 a new marker for monocytic human myeloid derived suppressor cells. Immunology 2012,

34. Ryzhov $S$, et al: Adenosinergic regulation of the expansion and immunosuppressive activity of CD11b+Gr1+ cells. J Immunol 2011, 187(11):6120-6129.

35. Greten TF, Manns MP, Korangy F: Myeloid derived suppressor cells in human diseases. Int Immunopharmacol 2011, 11(7):802-807.

36. Raychaudhuri B, et al: Myeloid-derived suppressor cell accumulation and function in patients with newly diagnosed glioblastoma. Neuro Oncol 2011, 13(6):591-599.

doi:10.1186/1746-6148-8-209

Cite this article as: Sherger et al:: Identification of myeloid derived suppressor cells in the peripheral blood of tumor bearing dogs. BMC Veterinary Research 2012 8:209. 